\title{
CONCERNING A CON JECTURE OF WHYBURN ON LIGHT OPEN MAPPINGS
}

\author{
BY LOUIS F. MCAULEY ${ }^{1}$ \\ Communicated by L. Zippin, January 25, 1965
}

Introduction. Some important and fundamental theorems in complex analysis are simple consequences of theorems in the theory of light open mappings for 2-manifolds. This rather complete theory is largely the work of G. T. Whyburn [9], [10], [11]. One theorem which includes the well-known theorems of Darboux [4] and Stoilow [8] is the following:

TheOREM (Whyburn). Suppose that $f$ is a light open mapping of a disk $A$ (topological 2-cell) onto a disk $B$ such that (a) $f($ Int $A$ ) $=$ Int $B$ and (b) $f \mid \operatorname{Bd} A$ is a homeomorphism of $\operatorname{Bd} A$ onto $\mathrm{Bd} B$. Then $f$ is a homeomorphism.

In his paper [12], Whyburn has conjectured that if in the above theorem each of $A$ and $B$ is a topological $n$-cell, then $f$ is a homeomorphism. This is an extremely difficult problem. One result of this announcement provides an affirmative answer for special cases of this conjecture. Church and Hemmingsen [1], [2], [3] have made significant contributions on related problems. Meisters and Olech [7] have some results for very special types of light open mappings; namely, either locally 1-1 maps or locally 1-1 maps except on discrete sets of a certain type.

Here, each mapping is continuous and each space is metric. A mapping $f$ of a space $X$ into a space $Y$ is light iff $f^{-1} f(x)$ is totally disconnected for each $x$ in $X$. And, $f$ is open iff for each $U$ open in $X, f(U)$ is open relative to $f(X)$.

Suppose that $f$ is a light mapping of a space $X$ into a space $Y$. We shall say that the singular set $S_{f}$ of $f$ is the set of points $x$ in $X$ such that $f$ is not locally $1-1$ at $x$; i.e., there is no set $U$ open in $X$ and containing $x$ such that $f \mid U$ is $1-1$. We consider here only mappings $f$ which preserve both the boundary and the interior of $X$ (both of which are assumed to be nonempty).

TheOREM 1. Suppose that $X$ is a compact subset of a metric space $M$, $\operatorname{Bd} X \neq 0$, Int $X \neq 0$, and $f$ is a light open mapping of $X$ into $M$ such that (1) $f(\operatorname{Int} X)=\operatorname{Int} f(X)$, (2) $f(\operatorname{Bd} X)=\operatorname{Bd} f(X)$, (3) the singular

1 The author carried out research on these and various other related problems at the University of Virginia where he held an ONR Research Fellowship, 1962-1963. 
set $S_{f}$ has the property that $f\left(S_{f}\right)$ does not contain a nonempty set open relative to $f(X),(4) f\left(S_{f}\right)$ does not separate $f(X)$, and (5) there exists a nonempty $U$ in $X$ open relative to $X$ such that $f \mid U$ is $1-1$ and $f^{-1} f(U)$ $=U$. Then $f$ is a homeomorphism.

This theorem is a generalization of theorems due to Meisters and Olech [6]. We use some techniques of theirs and also theorems from Whyburn's theory of light open mappings.

Let $P$ denote the set of all points $y$ in $f(X)$ such that $f^{-1}(y)$ is nondegenerate. Now, $f$ is a homeomorphism iff $P$ is empty. We shall show that $P$ is empty.

LEMMA 1. The set $P$ is open relative to $f(X)$ and contains a nonempty open set if $P$ is nonempty.

LEMma 2. The set $A=f\left(S_{f}\right) \cup P$ is closed and therefore compact.

Proof. Suppose that $y$ is a limit point of $A$ but $y \in f(X)-A$. Clearly, $y$ is a limit point of $P-f\left(S_{f}\right)$. Now, $f^{-1}(y)$ is a point $x$ and $f$ is locally 1-1 at $x$. Hence, there is a $n b h d N_{x}$ of $x$ such that $f \mid N_{x}$ is $1-1, f^{-1} f\left(N_{x}\right)=N_{x}$, and $f\left(N_{x}\right)$ is open relative to $f(X)$. This involves a contradiction.

Proof of Theorem 1. Suppose that $P$ is nonempty. It follows that $f\left(S_{f}\right) \cap$ Int $f(X)=f\left(S_{f} \cap\right.$ Int $\left.X\right)$. Now, $f\left(S_{f}\right)=f\left(S_{f} \cap \operatorname{Bd} X\right)$ $\cup f\left(S_{f} \cap \operatorname{Int} X\right)$. Also, $f(X)-f\left(S_{f}\right)=\left\{\left[f(X)-f\left(S_{f}\right)\right] \cap P\right\} \cup\{[f(X)$ $\left.\left.-f\left(S_{f}\right)\right] \cap[f(X)-P]\right\}$. Furthermore, $\left[f(X)-f\left(S_{f}\right)\right] \cap P$ is open relative to $f(X)$.

By Lemma 2, $f\left(S_{f}\right) \cup P$ is closed. Therefore, $B=f(X)-\left[f\left(S_{f}\right) \cup P\right]$ $=\left[f(X)-f\left(S_{f}\right)\right] \cap[f(X)-P]$ is open in $f(X)$. Both $B$ and $f(X)-f\left(S_{f}\right)$ are nonempty. Since $f(X)-f\left(S_{f}\right)$ is connected, $\left[f(X)-f\left(S_{f}\right)\right] \cap P=0$ and, consequently, $P=0$. We have a contradiction to our assumption that $f$ is not 1-1 and the theorem is proved.

Corollary 1. Suppose that $X$ is a compact proper subset of an $n$ manifold $M^{n}$ with Int $X \neq 0$. Furthermore, $f$ is a local homeomorphism of $X$ into $M^{n}$ such that (1) $f(X)$ is connected and (2) there is some set $U$ in $X$ open relative to $X$ such that $f^{-1} f(U)=U$ and $f \mid U$ is $1-1$. Then $f$ is a homeomorphism.

Corollary 2. Suppose that $X$ is a compact subset of $E^{n}$ with Int $X$ $\neq 0, X=$ closure of Int $X$, Int $f(X)$ is connected, and that $f$ is a light open mapping of $X$ into $E^{n}$ such that (1) $f \mid \operatorname{Int} X$ is locally 1-1, (2) $f($ Int $X)=\operatorname{Int} f(X),(3) \mathrm{Bd} f(X)=f(\operatorname{Bd} X)$, and (4) there is $U$ open relative to $X$ such that $f^{-1} f(U)=U$ and $f \mid U$ is 1-1. Then $f$ is a homeomorphism. 
Light open mappings on $n$-cells. Now, we are ready to give an affirmative answer to some special cases of Whyburn's conjecture. Consider the following theorems.

TheOREM 2. Suppose that $f$ is a light open mapping of an $n$-cell $A$ (unit ball in $E^{n}$ ) onto an n-cell $B$ (another unit ball) such that (1) $f^{-1} f(\operatorname{Bd} A)=\operatorname{Bd} A$, (2) $f(\operatorname{Bd} A)=\operatorname{Bd} B$, (3) dimension $f\left(S_{f}\right)<n$, (4) $B-f\left(S_{f}\right)$ is connected, and (5) there is $V$ in $B$ open relative to $B$ such that $f \mid f^{-1}(V)$ is 1-1. Then $f$ is a homeomorphism.

Theorem 2 is actually a corollary of Theorem 1 .

Theorem 3. Suppose that $f$ is a light open mapping of an n-cell $A$ (unit ball in $E^{n}$ ) onto an $n$-cell $B$ such that (1) $f^{-1} f(\mathrm{Bd} A)=\mathrm{Bd} A$, (2) $f(\operatorname{Bd} A)=\operatorname{Bd} B$, (3) $f \mid S_{f}$ is $1-1$, and (4) for each component $C$ of $B-f\left(S_{f}\right)$, there is $V$ in $C$ open relative to $B$ such that $f \mid f^{-1}(V)$ is 1-1. Then $f$ is a homeomorphism.

Proof. Clearly $f$ (Int $A)=$ Int $B$. Since $B$ is locally connected, there are at most a countable number of components $C_{1}, C_{2}, C_{3}, \cdots$, of $B-f\left(S_{f}\right)$. For each $i, f^{-1}\left(C_{i}\right)$ is connected. Denote it by $K_{i}$. Furthermore, $f\left(K_{i}\right)=C_{i}$.

Now, $f \mid K_{i}$ is locally 1-1. Also, $f\left(\bar{K}_{i}\right)=\bar{C}_{i}$ where $\bar{D}$ denotes the closure of $D$, and $f^{-1}\left(\bar{C}_{i}\right)=\bar{K}_{i}$. It follows that $f\left(\operatorname{Bd~} \bar{K}_{i}\right)=\operatorname{Bd} f\left(\bar{K}_{i}\right)$, $f\left(\right.$ Int $\left.\bar{K}_{i}\right)=\operatorname{Int} f\left(\bar{K}_{i}\right)$, and $f \mid \bar{K}_{i}$ is a light open mapping of $\bar{K}_{i}$ onto $\bar{C}_{i}$.

Apply Corollary 2 where $\bar{K}_{i}$ replaces $X$. Thus, $f \mid \bar{K}_{i}$ is a homeomorphism. Let $S=U_{i} \bar{K}_{i}$. Each point of $f\left(S_{f}\right)$ is a limit point of $B-f\left(S_{f}\right)$. Thus, $B=\bigcup_{i} \bar{C}_{i}$. It follows that $f(S)=B$ and that $f \mid S$ is a homeomorphism of $S$ onto $B$. We conclude that $S=A$ and that $f$ is a homeomorphism.

Questions. Suppose that condition (3), namely $f \mid S_{f}$ is $1-1$, is omitted from the hypothesis of Theorem 3. Is the resulting theorem true? Condition (3) may be weakened slightly as indicated in Theorem 4 below. Suppose that $f$ is a light open mapping of an $n$-cell $A$ onto an $n$-cell $B$. Does $f\left(S_{f}\right)$ contain an open set? This question has remained unsolved for several years (cf. [1], [2], [3]). If condition (3) that $f\left(S_{f}\right)$ fails to contain an open set is omitted from Theorem 1 , is the resulting theorem true?

$A$ generalization of Theorems 1 and 3 . In Theorem 1 , we require that $f\left(S_{f}\right)$ fail to separate $f(X)$ while in Theorem 3, we permit a separation but require that $f \mid S_{f}$ be 1-1. This may be weakened further.

Theorem 4. Suppose that $X$ is a compact subset of a metric space $M$, Bd $X \neq 0$, Int $X \neq 0$, and $f$ is a light open mapping of $X$ into $M$ such 
that (1) $f($ Int $X)=\operatorname{Int} f(X)$, (2) $f(\operatorname{Bd} X)=\operatorname{Bd} f(X)$, (3) if $p \in f\left(S_{f}\right)$ where $S_{f}$ is the singular set of $f$, then $p$ is in the boundary of some component of $f(X)=f\left(S_{f}\right)$, (4) if $C$ is a component of $f(X)-f\left(S_{f}\right)$, then $f \mid f^{-1}\left[\bar{C} \cap f\left(S_{f}\right)\right]$ is 1-1, and (5) for each component $K$ of $f(X)-f\left(S_{f}\right)$, there is $V$ in $K$ open relative to $f(X)$ such that $f \mid f^{-1}(V)$ is $1-1$. Then $f$ is a homeomorphism.

A proof of Theorem 4 may be obtained in a manner similar to that for Theorem 3.

\section{Bibliography}

0. K. Borsuk, Sur les groupes des classes de transformations continues, C. R. Acad. Sci. Paris 202 (1936), 1400-1403.

1. P. T. Church and E. Hemmingsen, Light open maps on n-manifolds, Duke Math. J. 27 (1960), 527-536.

2. - Light open maps on n-manifolds. II, Duke Math. J. 28 (1961), 607-624.

3. - Light open maps on n-manifolds. III, Duke Math. J. 30 (1963), 379-390.

4. G. Darboux, Lȩ̧ons sur la theorie general des surfaces et les applications géometriques $d u$ calcul infinitesimal, première partie, Paris, 1887, p. 173.

5. E. E. Floyd, Some characterizations of interior maps, Ann. of Math. 51 (1950), 571-575.

6. W. Hurewicz and H. Wallman, Dimension theory, Princeton Univ. Press, Princeton, N. J., 1948.

7. G. H. Meisters and C. Olech, Locally one-to-one mappings and a classical theorem on schlicht functions, Duke Math. J. 30 (1963), 63-80.

8. S. Stoillow, Sur un theorème topologique, Fund. Math. 13 (1929), 186-194.

9. G. T. Whyburn, Analytic topology, Amer. Math. Soc. Colloq. Publ. Vol. 28, Amer. Math. Soc., Providence, R. I., 1942.

10. - Topological analysis, Princeton Univ. Press, Princeton, N. J., 1958.

11. - Open mappings on 2-dimensional manifolds, J. Math. Mech. 10 (1961), 181-198.

12. - An open mapping approach to Hurwitz's theorem, Trans. Amer. Math. Soc. 71 (1951), 113-119.

Rutgers, The State University 